\title{
Fatores relacionados ao manejo clínico e educacional do paciente em período pré e pós-transplante cardíaco: revisão integrativa
}

RESUMO | Objetivo: Analisar as recomendações de condutas terapêuticas e educacionais na assistência ao paciente em período pré e pós-transplante cardíaco. Métodos: Trata-se de revisão integrativa baseada na estratégia PICO, realizada com 12 artigos gerados através de pesquisa nas bases de dados LILACS; MEDLINE; BDENF; IBECS; BINACIS e COLECIONASUS em agosto de 2019. Resultados: É recomendado a atuação da equipe multidisciplinar especializada em transplante e a educação em saúde. Maior grau de instrução, melhor condição cognitiva e altos níveis de satisfação social são preditivos de aumento nos anos de sobrevida pós-transplante. Os principais aspectos a serem trabalhados são: ação da insuficiência cardíaca e seu impacto no organismo, tratamentos disponíveis, período de recuperação, rotinas de exames e acompanhamento. Conclusão: Diante da complexidade do tema faz-se necessário uma equipe assistencial especializada e a reflexão sobre a inclusão do cardiopata e familiares no processo. O apoio psicológico gera melhores resultados e adesão ao tratamento.

Palavras-chaves: Transplantados; Insuficiência Cardíaca; Transplante de Coração; Educação de Pacientes Como Assunto; Assistência ao Paciente.

\begin{abstract}
Objective: To analyze the recommendations for therapeutic and educational approaches in patient care in the preand post-heart transplant period. Methods: This is an integrative review based on the PICO strategy, carried out with 12 articles generated thrçough research in the LILACS databases; MEDLINE; BDENF; IBECS; BINACIS and COLECIONASUS in August 2019. Results: The work of a multidisciplinary team specialized in transplantation and health education is recommended. A higher level of education, a better cognitive condition and high levels of social satisfaction are predictive of an increase in the years of post-transplant survival. The main aspects to be worked on are: heart failure action and its impact on the organism, available treatments, recovery period, exam routines and follow-up. Conclusion: In view of the complexity of the theme, a specialized assistance team and reflection on the inclusion of cardiopath and family members in the process is necessary. Psychological support generates better results and treatment adherence.
\end{abstract}

Keywords: Transplanted Patients; Heart Failure; Heart Transplantation; Patient Education As Topic; Patient Assistance.

RESUMEN | Objetivo: Analizar las recomendaciones de abordajes terapéuticos y educativos en la atención al paciente en el período pre y postrasplante cardíaco. Métodos: Se trata de una revisión integradora basada en la estrategia PICO, realizada con 12 artículos generados a través de la investigación en las bases de datos LILACS; MEDLINE; BDENF; IBECS; BINACIS y COLECIONASUS en agosto de 2019. Resultados: Se recomienda el trabajo de un equipo multidisciplinario especializado en trasplantes y educación para la salud. Un mayor nivel de educación, una mejor condición cognitiva y altos niveles de satisfacción social son predictores de un aumento en los años de supervivencia postrasplante. Los principales aspectos a trabajar son: acción de la insuficiencia cardíaca y su impacto en el organismo, tratamientos disponibles, período de recuperación, rutinas de exploración y seguimiento. Conclusión: Dada la complejidad del tema, es necesario un equipo asistencial especializado y la reflexión sobre la inclusión del cardiópata y familiares en el proceso. El apoyo psicológico genera mejores resultados y adherencia al tratamiento. Palabras claves: Pacientes transplantados; insuficiencia cardíaca; trasplante cardía educación del paciente como tema; Asistencia al paciente.

\section{Gleicilaine Alves de Souza}

Enfermeira da Equipe Multiprofissional de Atenção Domiciliar do município de Santa Luzia/MG. Especialista em Saúde Cardiovascular pelo Programa de Residência Multiprofissional do Hospital das Cçlínicas da Universidade Federal de Minas Gerais (HC-UFMG).

ORCID: 0000-0002-9303-4298

\section{Karla Cordeiro Gonçalves}

Enfermeira do HC-UFMG. Mestre em Ciências Aplicadas à Saúde do Adulto pela UFMG. Especialista em cardiologia pelo Hospital das Clínicas da Faculdade de Medicina da Universidade de São Paulo (HC-FMUSP). ORCID: 0000-0002-5123-9308

\section{Salete Maria de Fátima Silqueira}

Enfermeira. Dra em Saúde Pública pela Universidade de São Paulo. Tutora da residência multiprofissional em Saúde Cardiovascular do HC-UFMG.

ORCID: 0000-0002-4248-7107

Recebido em: 30/12/2020

Aprovado em: 26/01/2021
INTRODUÇÃO

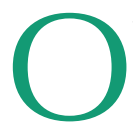
transplante cardíaco (TC) consiste em tratamento para pacientes com insuficiência cardíaca (IC) grave que não respondem à terapia com fármacos e outros procedimentos $^{(1,2)}$. Com o avanço da tecnologia e tratamentos das miocardiopatias, a incidência da IC tem crescido nas últimas décadas, além disso o aumento da expectativa de vida e a melhoria do prognóstico do infarto agudo do miocárdio contribui para que o número de candidatos à realização de TC se eleve a cada ano ${ }^{(2,3)}$. 
Para recomendar o TC é preciso considerar aspectos que afetam o sucesso do procedimento e reabilitação, como: estágio e avanço da doença, impacto na qualidade de vida, fatores de risco e expectativa de adesão ao tratamento pós-transplante ${ }^{(2,4,5)}$

O processo saúde-doença, seja ele cardíaco ou não, requer do indivíduo uma adaptação à sua nova condição, algumas vezes temporária, outras permanente. O manejo de qualquer enfermidade visa cura e/ou qualidade de vida, oferecendo conforto e, quando possível, independência ${ }^{(6)}$. Para que este propósito seja atingido no cardiopata, é necessário que os profissionais estejam dispostos a atuar de maneira qualificada, além de promover a educação do paciente com as informações necessárias sobre o tratamento, procedimentos e acompanhamento pós alta hospitalar ${ }^{(7)}$.

O transplante cardíaco não resulta em cura absoluta, ele faz parte de um processo que visa melhora da qualidade de vida e, para isso, o indivíduo transplantado terá que se adaptar a certas restrições e conviver com outras condições de saúde ${ }^{(4)}$. Assim, é preciso compreender a expectativa que o candidato ao TC possui para se utilizar abordagem adequada às orientações ${ }^{(7)}$. Até a chegada do coração compatível o paciente recebe cuidados que envolvem terapia medicamentosa otimizada para controle da progressão da doença, este é um momento oportuno para sanar dúvidas acerca das condições de vida e cuidados que deverá ter depois da realização da cirurgia ${ }^{(8,9)}$.

Devido à complexidade do TC são necessárias adaptações à rotina para manutenção do bem-estar e prevenção de complicações, isto irá alterar o cotidiano e impactar nas escolhas pessoais, perfil e modo de vida, o paciente pode apresen-

\section{Figura 1: Percurso para busca e seleção dos estudos nas bases de dados.}
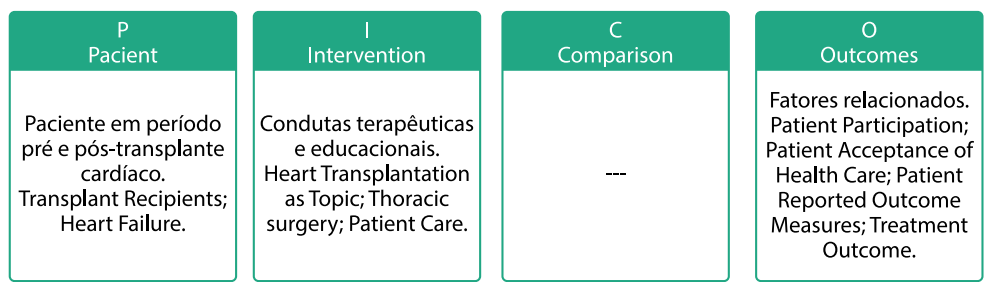

Figura 2 - Percurso de seleção dos estudos

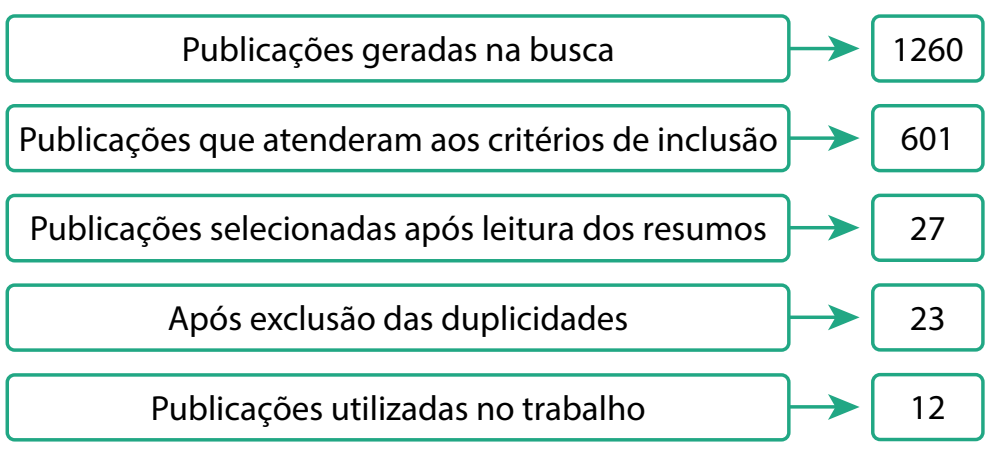

tar dificuldades em lidar com este processo e comprometer assim o tratamento ${ }^{(10)}$.

Diante do exposto, a presente pesquisa objetivou analisar as recomendações de condutas terapêuticas e educacionais na assistência ao paciente neste período.

\section{MÉTODOS}

Trata-se de revisão integrativa, uma análise ampla da literatura para adquirir conhecimento profundo baseado em outros artigos e guiado através das etapas: 1) definição da questão de pesquisa, 2) busca na literatura e seleção de artigos, 3) extração dos dados dos estudos, 4) avaliação das produções, 5) interpretação dos resultados e 6) síntese e apresentação do conhecimento $^{(11)}$.

A questão foi elaborada através do método PICO (acrônimo de patient, intervention, comparison, outcomes). O uso dessa estratégia possibilita a identificação de termos-chave para a localização de estudos relevantes ${ }^{(12)}$. O primeiro elemento da estratégia referiu-se a paciente em período pré e pós-transplante cardíaco (P), o segundo (I) nas condutas terapêuticas e educacionais. Dependendo da revisão, não se emprega todos os elementos da estratégia PICO, neste artigo o terceiro tópico, a comparação (C), não foi utilizada. O quarto elemento refere-se aos fatores que se relacionam à assistência (O). A Figura 1 traz a descrição dos componentes da pergunta e descritores definidos.

Após a definição dos descritores foi realizado o cruzamento entre eles juntamente com sinônimos e versões em inglês e espanhol utilizando-se os operadores booleanos "AND" e "OR". Os cruzamentos foram inseridos no portal da Biblioteca Virtual em Saúde nas bases de dados MEDLINE, LILACS, BDENF, IBECS, BINACIS e COLECIONASUS em agosto de 2019. Os critérios de inclusão foram: estudos em português, inglês e espanhol, completos e disponíveis online. Não foi estabelecido limite temporal devido à escassez de artigos sobre o 
tema. A Figura 2 descreve o percurso de seleção dos estudos.

Foram extraídos dos estudos os seguintes dados: identificação do artigo, instituição sede do estudo, tipo de publicação, características metodológicas do estudo e avaliação do rigor metodológico, tais informações foram definidas através do uso de um instrumento estruturado e validado por Ursi(13) (2005). A validação da pesquisa foi realizada por dois pesquisadores a fim de aumentar a confiabilidade do estudo.

Foi realizada leitura analítica dos estudos identificando os pontos chave para hierarquização e síntese das ideias.

\section{RESULTADOS}

Analisou-se 12 pesquisas que atenderam aos critérios de inclusão, estas serão apresentadas mais detalhadamente no Quadro 1 que relaciona nome do trabaIho e dos autores, ano e país de publicação e objetivos do estudo.

Quadro 1 - Relação dos artigos selecionados para o estudo.

n

Título do artigo

Autor

Objetivo

Managing patients with heart failure all the way to a heart transplant

Heart transplant centers with multidisciplinary team show a higher

2 level of chronic illness management e Findings from the International BRIGHT Study

Learning to Live Again The Role

3 of Education in Heart Transplant Recipients

4 Vivenciando o transplante cardíaco: a perspectiva dos pacientes

PIROZZI, R.(14)

\begin{tabular}{|c|}
\hline Ano/país \\
2018 Estados \\
Unidos
\end{tabular}

Descrever o manejo de pacientes com insuficiência cardíaca até o transplante

Explorar a proporção dos centros de transplante cardíaco que tem uma equipe multidisciplinar e descrever a composição da equipe; avaliar a relação entre multidisciplinar e o nível de gestão de doenças crônicas em centros transplante cardíaco.

\begin{tabular}{|c|c|c|c|}
\hline 4 & perspectiva dos pacientes & MLA(17) & 2006 Brasil \\
\hline 5 & $\begin{array}{l}\text { Health literacy in patients referred } \\
\text { for transplant: do patients have the } \\
\text { capacity to understand? }\end{array}$ & $\begin{array}{c}\text { MILLER-MATERO, LR et } \\
\text { al.(18) }\end{array}$ & $\begin{array}{c}2015 \text { Estados } \\
\text { Unidos }\end{array}$ \\
\hline
\end{tabular}
com base nas descrições dos pacientes.

Estimar a prevalência de baixo nível de alfabetização de saúde, determinar as características de pacientes que podem ter um risco aumentado para baixos níveis e capacidades limitadas; e investigar a relação entre a capacidade de leitura, habilidade matemática, e funcionamento cognitivo.

Discutir a experiência do Instituto do Coração (InCor) de São Paulo, na implementação do conceito

6 0 "Heart Team" para transplante cardíaco: organização e funcionamento

SEGURO, LFBC et al.(19)

2014 Brasil

de centros transplantadores com uma equipe multidisciplinar trabalhando exclusivamente numa ampla rede que envolve o transplante cardíaco

Psychological assessment of patients

7 undergoing cardiac transplant in a teaching hospital (2004 to 2012)

8 Percepção de pacientes sobre o período pré-operatório de Cirurgia cardíaca

CUNHA, SS et al.(20)

2014 Brasil

Analisar as avaliações psicológicas de pacientes com insuficiência cardíaca aguardando transplante cardíaco.

Conhecer qual a percepção dos pacientes em período pré-operatório de cirurgia cardiovascular sobre seu processo saúde-doença, bem como sobre o procedimento cirúrgico.

Demographic, Psychosocial, and

9 Behavioral Factors Associated With Survival After Heart Transplantation

CAMPONOGARA, S. et al(21)

2012 Brasil Educación al paciente y la familia en un programa de trasplante, experiencia en CLC / Family and patient education program in transplant: clínica las condes experience

2013 Estados Unidos

FARMER, AS et al.(22)

HERZOG, CO.(23)

2010 Chile

Determinar fatores demográficos, comportamentais e de qualidade de vida associados à mortalidade 5 a 10 anos após o transplante cardíaco.

Apresentar o componente educacional entregue aos pacientes durante o processo de transplante, desde a admissão ao programa até a alta e o acompanhamento ambulatorial pós-transplante.

2016 Brasil
Investigar a experiência do transplante cardíaco. 


12 \begin{tabular}{c|c|}
\hline 12 & $\begin{array}{c}\text { Effect of Preoperative Education on } \\
\text { Postoperative Outcomes Among Pa- } \\
\text { tients Undergoing Cardiac Surgery: A } \\
\text { Systematic Review and Meta-Analysis }\end{array}$ \\
\hline
\end{tabular}

Quanto ao idioma de origem, 07 foram escritos em inglês $\left({ }^{14-16,18,20,22,25}\right), 04$ em português ${ }^{(17,19,21,24)}$ e 01 em espanhol ${ }^{(23)}$. O nível de evidência varia de 1 a 6 , predominando o nível 4 (42\%), conforme categorização elaborada para avaliar pesquisas, baseada na qualificação da Agency for Healthcare Research and Quality (AHRQ) dos Estados Unidos (EUA) ${ }^{(26)}$.

Em relação às categorias profissionais dos autores destacou-se o profissional en- fermeiro, presente em $66,7 \%$ dos artigos, médicos estão presentes em 06 publicações. Psicólogos atuaram em duas produções, biomédico e pedagogo em uma pesquisa cada.

Todas as produções brasilei$\operatorname{ras}^{(17,19-21,24)}$ tiveram como cenário de pesquisa instituições públicas de saúde, já os artigos internacionais ${ }^{(14,16,18,22,23,25)}$ utilizaram-se apenas de hospitais privados, com exceção de uma investigação produzida

\section{Gráfico 1 - Distribuição dos estudos por país de origem.}

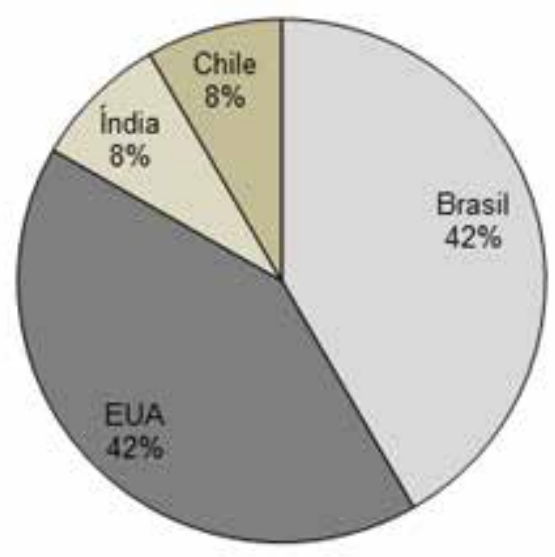

Gráfico 2 - Composição da equipe multiprofissional indicada pelas publicações.

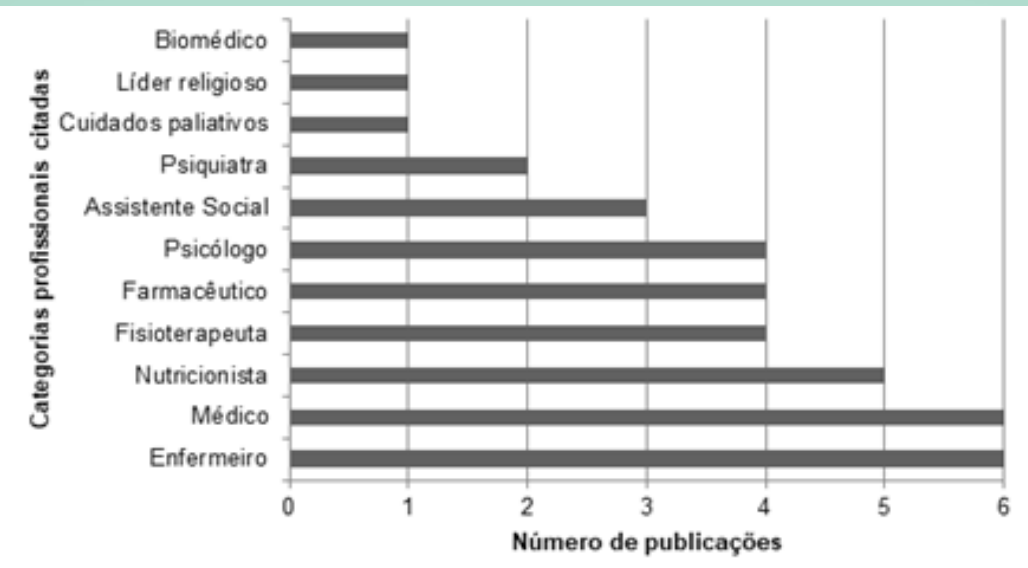

Avaliar o efeito da educação pré-operatória sobre os resultados pós-operatórios entre os pacientes submetidos à cirurgia cardíaca. nos Estados Unidos ${ }^{(15)}$ que inclui centros de transplante da América e Europa, porém não relata o quantitativo de organizações públicas e privadas incluídas na pesquisa.

Nove estudos ${ }^{(14-17,19,20,23-25)}$ ressaltam a eficácia da equipe multiprofissional na busca pela qualidade de vida e diminuição da mortalidade, $04 \operatorname{artigos}^{(14,16,19,23)}$ apontam o enfermeiro como pessoa indicada para função de coordenador da equipe de transplante.

Uma pesquisa que avaliou a presença de equipe multidisciplinar em diversos países relata que apenas o Brasil e EUA possuem legislação que obrigam a atuação da equipe multiprofissional no acompanhamento desses pacientes. Ainda assim os autores perceberam que, dos 03 centros transplantadores brasileiros que participaram do estudo, 01 não apresentava atuação interdisciplinar especializada, (os autores consideraram equipe multiprofissional aquela composta por no mínimo: um médico, um enfermeiro e um outro profissional da saúde). Foi constatada a falta do psicólogo, assistente social, farmacêutico e fisioterapeuta em pelo menos uma instituição brasileira participante ${ }^{(15)}$.

Seis estudos discutiram sobre as categorias profissionais que devem compor a equipe de transplante $\mathrm{e}^{(14-16,19,23,25)}$. O Gráfico 2 expõe o quantitativo de indicações por categoria profissional.

Estudos sociodemográficos concluíram que maior grau de instrução, melhor condição cognitiva e altos níveis de satisfação social são preditivos de aumento nos anos de sobrevida pós-transplante. Pacientes com alfabetização em saúde limitada tendem a ter maior taxa de hospitalização e risco de mortalidade ${ }^{(18,22)}$.

Sobre as condutas específicas no gerenciamento da terapia, deve-se realizar o manejo da doença promovendo con- 
trole dos sintomas e abordagem educacional junto ao paciente acerca da ação da IC e seu impacto no organismo, tratamentos indicados, procedimento cirúrgico, reabilitação, hábitos alimentares, informações farmacológicas, atividade física, sinais e sintomas de alerta, rotinas de exames e acompanhamento ${ }^{(14-25)}$.

Quanto ao reconhecimento da limitação, 07 estudos recomendaram a realização de pesquisas para complementar o tema e atingir o patamar de maior nível de evidência dos achados ${ }^{(15,17,18,20,21,24,25)}$. Três artigos indicaram lacunas na literatura quanto à avaliação da experiência subjetiva do paciente e a eficácia da educação em saúde ${ }^{(17,18,25)}$.

\section{DISCUSSÃO}

Em resposta à questão norteadora, a literatura recomenda o manejo através de uma equipe multiprofissional especializada em transplante ${ }^{(14-17,19,20,23-25)}$, o tratamento deve buscar melhora da qualidade de vida, diminuição da mortalidade, controle de sintomas, redução de fatores de risco e inclusão do paciente em fila de transplante antes da piora clínica grave, a fim de garantir estabilidade hemodinâmica no procedimento ${ }^{(14,19)}$.

No Brasil a maior parte dos transplantes ocorre em pacientes hospitalizados e instáveis, muitos já dependentes do uso do balão intra-aórtico, diferente dos EUA e países europeus, que utilizam dispositivos de assistência circulatória mais avançados que promovem controle dos agravos, assim grande parte dos pacientes são encaminhados para o transplante com melhor estabilidade, o que favorece o sucesso do tratamento e taxa de sobrevivência ${ }^{(19)}$.

Diante da vinculação do desfecho positivo com a eficácia da educação em saúde e assistência psicossocial ${ }^{(14-25)}$ é preciso promover o aprendizado do paciente de acordo com seu nível de escolaridade e estado cognitivo ${ }^{(18,22)}$, quanto a este último ressalta-se que o avanço da falência do órgão, pode comprometer o grau de entendimento do portador da doença, uma vez que a hipoperfusão de tecidos e o contexto de afastamento social, causado pela hospitalização, afeta a cognição. Assim, deve-se avaliar a capacidade de entendimento do paciente diariamente, atentando-se para a condição mental no momento das orientações ${ }^{(18)}$.

Os objetivos estabelecidos nos estudos indicam a relevância da investigação da percepção do indivíduo em tratamento. Pesquisas dessa natureza oferecem subsídios importantes para conhecer as necessidades, a partir da visão própria do paciente ${ }^{(17)}$.

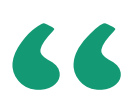

porcionar um ambiente onde o paciente sinta-se à vontade para sanar suas dúvidas e expor seus anseios e expectativas ${ }^{(21)}$.

Percebe-se numerosas recomendações acerca da importância da atuação da equipe multiprofissional ${ }^{(14-17,19,20,21-25)}$. Entre os tópicos abordados destaca-se: identificação em tempo hábil dos casos refratários ao tratamento, avaliação biopsicossocial, cuidados do paciente em fila buscando manter sua estabilidade, assistência pós-operatória para reabilitação, reinserção na rotina de vida e educação visando autonomia e autocuidado ${ }^{(19)}$.

Apesar das evidências acerca do impacto da atuação multiprofissional, é possível perceber em muitos locais a ausência de alguns profissionais ${ }^{(15)}$. Esse fato pode prejudicar a percepção do indivíduo e familiares quanto à assistência, pois pacientes atendidos por equipe multiprofissional relatam maior satisfação com sua recuperação, o que impacta na adesão ao tratamento e, consequentemente, na taxa de sobrevida ${ }^{(17,21)}$.

O apoio psicológico é fundamental $^{(14-16,20,25)}$, pacientes transplantados cardíacos falaram sobre o significado de suas relações com os profissionais de saúde e ressaltaram o vínculo construído durante o tratamento ${ }^{(17)}$. Há ainda a simbologia a qual o coração está ligado culturalmente. Muitas pessoas atribuem a este órgão a função de detentor de sentimentos e emoções e a troca do mesmo pode representar um impacto emocional ${ }^{(17,21,24)}$. Estudos constataram pensamentos de culpa em transplantados causados pelo sentimento de estar vivendo em virtude da morte de outro ${ }^{(17,24)}$. O preconceito também é citado, o paciente pode perceber a discriminação ao retornar para o cotidiano, especialmente no trabalho ${ }^{(24)}$.

A presença de enfermeiros na autoria de $66 \%$ dos estudos justifica-se pela grande participação nos cuidados diários e na importância que este profissional atribui ao aspecto subjetivo, percepção e sentimentos do cliente sob sua assistência, tudo isso através da proximidade com o paciente e sua família, já que permane- 
ce durante todo o período de internação junto a eles, e, na maioria das vezes, é o profissional a quem o paciente sente-se mais à vontade para expor seus medos, dúvidas e expectativas ${ }^{(19)}$.

O coordenador da equipe de transplante irá avaliar o cumprimento das ações, realizar as recomendações de autocuidado, promover educação em saúde, desenvolver estratégias de envolvimento de toda a equipe multiprofissional nos cuidados necessários e avaliar a eficácia da assistência prestada através dos resultados obtidos. 04 estudos consideram o enfermeiro como pessoa qualificada para assumir esta função, isto porque se trata de profissional próximo ao paciente, com facilidade de estabelecimento de vínculo afetivo e expressão de $\operatorname{confiança}^{(14,16,19,23)}$. Outra pesquisa destaca o psicólogo como profissional capacitado a exercer essa atividade ${ }^{(18)}$.

Sobre as pesquisas brasileiras serem todas em instituições públicas justifica-se pelas políticas de saúde no país que determinam a realização do transplante de órgãos somente em estabelecimentos de saúde e médico-cirúrgicas de remoção e transplante permitidos através da gestão nacional do Sistema Único de Saúde ${ }^{(27)}$.

\section{CONCLUSÃO}

O manejo clínico e educacional do paciente pré e pós-transplante cardíaco apresenta grande complexidade e requer da equipe um conhecimento específico acerca deste tipo de procedimento e con- dutas terapêuticas. A IC avançada traz ao paciente sentimentos de medo, angústia e dúvidas, por isso é necessária a reflexão sobre a inclusão do cardiopata e familiares no processo de tratamento. $\mathrm{O}$ apoio psicológico é indispensável e o aspecto subjetivo precisa ser considerado a fim de gerar aproximação entre profissionais e pacientes, possibilitando melhor adesão ao tratamento.

Os resultados deste estudo podem subsidiar discussões e reflexões acerca da importância de uma assistência integral e da qualidade das orientações fornecidas, possibilitando assim mudanças paradigmáticas na assistência.

Como limitação notou-se a presença de pesquisas com lacunas na elucidação dos métodos e objetivos.

\section{Referências}

1. Pio FSCG, et al. Assistência de enfermagem no transplante cardíaco: revisão integrativa. Rev enferm UFPE. 2016 Maio; 10(5):1857-65.

2. Bacal F, et al. $3^{a}$ Diretriz Brasileira de TC. Arq Bras Cardiol. 2018; 111(2):230289.

3. Fiorelli $\mathrm{A}$; Oliveira $\mathrm{J}$; Stolf $\mathrm{N}$. Transplante cardíaco. revistadc 6set.2009;88(3):123-37.

4. Pfeifer PM, Ruschel PP. Preparo psicológico: a influência na utilização de estratégias de enfrentamento pós-transplante cardíaco. Rev. SBPH. 2013 Dez; 16(2): 153-165.

5. Costa K, et al. A importância das orientações de enfermagem no cuidado ao paciente submetido à cirurgia cardíaca: revisão integrativa. Rev FUG, v.9, n.2, jul-dez. 2015, p.3.

6. Vianna, LAC. Processo saúde doença. UNIFESP. Versão 2.13 de abril de 2012. 7. Goncalves KKN, Silva Jl, Gomes ET, Pinheiro LLS, Figueiredo TR, Bezerra SMMS. Anxiety in the preoperative period of heart surgery. Rev Bras Enferm. 2016:69(2):374-80.

8. Mangini S, et al. Transplante cardíaco: revisão. Einstein/SP. 2015 Jun; 13(2): 310-318.

9. Baggio MA, Teixeira A, Portella MR. Pré-operatório do paciente cirúrgico cardíaco: a orientação de enfermagem fazendo a diferença. Rev Gaúcha Enferm. 2001;22(1):122-39.

10. Vasconcelos AG, Pessoa VL, Menezes F, Florêncio R, Frota MX. Repercussions on the daily living of post-heart transplantation patients. Acta Paul Enferm. 2015;28(6):573-9.

11. Mendes K, Silveira R, Galvão C. Revisão integrativa: método de pesquisa para incorporação de evidências na saúde e na enfermagem. Texto cont-enferm. 2008 Dec; 17(4): 758-764.

12. Santos C, Pimenta C, Nobre M. A estratégia PICO para a construção da pergunta de pesquisa e busca de evidências. Rev. Latino-Am. Enferm. 2007 Jun; 15(3): 508-511.

13. Ursi ES. Prevenção de lesões de pele no perioperatório: revisão integrativa da literatura. [dissertation]. Ribeirão Preto: Universidade de São Paulo, Escola de Enfermagem de Ribeirão Preto; 2005.

14. Pirozzi R; Managing patients with heart failure all the way to a heart transplant. Pittsburgh, Pensilvânia. Crit Care Nurs Q. Vol. 41, № 4, p369-375. 2018.

15. Cajita MI, et al; Heart transplant centers with multidisciplinary team show a higher level of chronic illness management e Findings from the International BRIGHT Study. Baltimore, Maryland. Heart \& Lung, 1-6, 2017.

16. Mohney K; Learning to Live Again The Role of Education in Heart Transplant Recipients. Pittsburgh, Pensilvânia. Crit Care Nurs Q. Vol. 41, № 4, pag 389-393. 2018.

17. Stolf NG; Sadala MLA; Os significados de ter o coração transplantado: a experiência dos pacientes. São Paulo, SP. Braz J Cardiovasc Surg 2006; 21(3): 314-323.

18. Miller ML; Hyde N; Abouljoud M; Health literacy in patients referred for transplant: do patients have the capacity to understand? Detroit. Clin Transplant 2015: 29: 336-342.

19. Seguro LFBC et al; 0 "Heart Time" para transplante cardíaco: organização e funcionamento. Rev Soc Cardiol. São Paulo. 2014;24(3):54-61.

20. Cunha SS; et al; Psychological assessment of patients undergoing cardiac transplant in a teaching hospital (2004 to 2012). SP. Rev Bras Cir Cardiovasc 2014:29(3):350-4

21. Campognara $S$, et al; Percepção de pacientes sobre o período pré-operatório de cirurgia cardíaca. Santa Maria, RS. Rev. Min. Enferm.;16(3): 382-390, jul./set. 2012.

22. Farmer SA, et al; Demographic, Psychosocial, and Behavioral Factors Associated With Survival After Heart Transplantation. Chicago. Ann Thorac Surg 2013;95:876-83.

23. Herzog C; Educación al paciente y la familia en un programa de trasplante, experiencia en CLC. Chile. Rev. Med. Clin. Condes - 201021 (2) 293-299.

24. Sadala MLA; Stolf NAG; Bicudo MAV; Transplante cardíaco (TC) a experiência do portador da Doença de Chagas. Rev. esc. enferm. USP. 2009, vol.43, n.3, pp.588-595.

25. Ramesh C; et. al; Effect of Preoperative Education on Postoperative Outcomes Among Patients Undergoing Cardiac Surgery: A Systematic Review and Meta-Analysis. Journal of perianesthesia Enfermagem, Vol -, Não - (-), 2016: pp 1-12.

26. Stetler $C B$, et al. Utilization-focused integrative reviews in a nursing service. Appl Nurs Res.1998;11(4):195-206

27. BRASIL. Lei n.9434, de 04 de fevereiro de 1997. Dispõe sobre a remoção de órgãos, tecidos e partes do corpo humano para fins de transplante e tratamento. 